\title{
Novel and facile synthesis of furanodictines A and B based on transformation of
}

\section{2-acetamido-2-deoxy-D-glucose into 3,6-anhydro hexofuranoses}

\author{
Makoto Ogata, ${ }^{a, b}$ Takeshi Hattori, ${ }^{a}$ Ryota Takeuchi, ${ }^{a}$ and Taichi Usui ${ }^{a}, b,{ }^{*}$
}

${ }^{\text {a }}$ Department of Bioscience, Graduate School of Science and Technology, Shizuoka University, Ohya 836, Suruga ward, Shizuoka 422-8529, Japan

${ }^{b}$ Department of Applied Biological Chemistry, Faculty of Agriculture, Shizuoka University, Ohya 836, Suruga ward, Shizuoka 422-8529, Japan

* Corresponding author. Fax: +81-54-238-4873; e-mail: actusui@agr.shizuoka.ac.jp 


\begin{abstract}
A

novel

synthesis

of

furanodictines

A

[2-acetamido-3,6-anhydro-2-deoxy-5-O-isovaleryl-D-glucofuranose

(1)] and

$\mathrm{B}$

[2-acetamido-3,6-anhydro-2-deoxy-5-O-isovaleryl-D-mannofuranose

(2)] are described

starting from 2-acetamido-2-deoxy-D-glucose (GlcNAc). The synthetic protocol is based on deriving

the

epimeric

bicyclic

3,6-anhydro

sugars

[2-acetamido-3,6-anhydro-2-deoxy-D-glucofuranose

(4)

2-acetamido-3,6-anhydro-2-deoxy-D-mannofuranose (5)] from GlcNAc. The reaction with borate upon heating led to a facile transformation of GlcNAc into the desired epimeric 3,6-anhydro sugars. The hydroxy groups of $\mathrm{C}_{5}$ of the 3,6-anhydro compounds 4 and $\mathbf{5}$ were regioselectively esterified with the isovaleryl chloride to complete the synthesis of furanodictines A and B, respectively. The targets $\mathbf{1}$ and $\mathbf{2}$ were synthesized in only two-steps requiring no protection/deprotection.
\end{abstract}

Keywords: $\quad$ 2-Acetamido-2-deoxy-D-glucose; Bicyclic $\quad$ 3,6-anhydroaminosugar; 3,6-anhydrohexofuranose; Furanodictines A and B; GlcNAc transformation. 


\section{Introduction}

Oshima et al isolated the 3,6-anhydro amino sugar derivatives, furanodictines A (1) and B (2) from the metabolic extract of the fruiting body of the cellular slime mold Dictyostelium discoideum (Fig. 1). ${ }^{1}$ Both these compounds display potent neuronal differentiation activity in rat PC-12 cells. ${ }^{1,2}$ The absolute configurations of furanodictines A and B were confirmed by asymmetric syntheses of $\mathbf{1}$ and $\mathbf{2}$, which possess a 3,6-anhydro hexofuranose carbon skelton corresponding to 4 and 5, respectively. Furanodictines A (1) and B (2) are the first examples of amino sugars with an interesting bicyclic bis-tetrahydrofurofuran structure. The structural complexity, coupled with the potent biological activity, make $\mathbf{1}$ and $\mathbf{2}$ attractive targets for

total synthesis. ${ }^{3-5}$ Although total synthesis has been achieved, these synthetic procedures generally require multi-step pathways and are unsatisfactory for obtaining the target compounds in reasonable yield. There is an urgent need to prepare large quantities of such pharmacophore-containing compounds from easily available raw materials by simple methods. Here a simple synthetic strategy for $\mathbf{1}$ and $\mathbf{2}$ starting from readily available GlcNAc was designed and executed.

\section{Results and discussion}

\subsection{Transformation of GIcNAc into hexofuranoses}

The reaction was initially carried out at a concentration of GlcNAc $(100 \mathrm{mM})$ in borate solution $(\mathrm{pH} 7.0)$ at $100^{\circ} \mathrm{C}$ for $2 \mathrm{~h}$. The reaction mixture was applied to a column of charcoal-Celite with a linear gradient of ethanol as shown in Fig. 2. The chromatogram showed five fractions (F-1 F-5), numbered according to the order of elution. Each fraction was collected and analyzed separately. Fraction 1 contained GlcNAc and 2-acetamido-2-deoxy-D-mannose (ManNAc) in proportion of $2: 1$, respectively, in a yield of 10\%. Fraction 2 was not identified. Fractions 3, 4 and 5 were obtained in actual yields of 9.8 , 10.2 and $36 \%$, respectively, and identified as the respective unsaturated derivative 3 (F-5) and two epimeric 3,6-anhydro derivatives 4 (F-4) and 5 (F-3). In the Morgan-Elson reaction, Chromogen I (2-acetamido-2,3-dideoxy-D-erythro-hex-2-enofuranose, 3) is known to be the 
major chromogen formed by alkali treatment of GlcNAc under the conditions used by Kuhn and Kruger. ${ }^{6,7}$ Sinay et al have established that the major Chromogen I of the Morgan-Elson reaction is the structure of $\mathbf{3}$, which was derived in four steps from ManNAc. ${ }^{8}$ Compound $\mathbf{3}$ was produced in preference to epimeric 3,6-anhydro forms 4 and 5. ${ }^{9}$ Derevitakaya et al have reported that the epimeric $\mathbf{4}$ and $\mathbf{5}$ are formed in low yields during the alkaline degradation of GlcNAc, based on the Morgan-Elson reaction. Compounds 4 and 5 are formed in a $1: 1$ ratio during the entire course of the reaction. These results indicated that about two-thirds of GlcNAc as starting material are converted into a series of hexofuranose derivatives. The structures of these compounds were evaluated by ${ }^{1} \mathrm{H}$ and ${ }^{13} \mathrm{C}$ NMR analyses in $\mathrm{D}_{2} \mathrm{O}$ solution, consistent with published data. ${ }^{8} 10$ In addition, ESI-MS analysis was also carried out on each fraction. The same molecular ion at $m / z 226$ was obtained for 3, 4 and $\mathbf{5}$ arising from the $[\mathrm{M}+$ $\mathrm{Na}]^{+}$ion of GlcNAc with loss of mass corresponding to a water molecule. As a result, we established a facile transformation of GlcNAc into $\mathbf{4}$ and $\mathbf{5}$, which is useful for synthesis of furanodictines A and B.

\subsection{Time-course analysis of GIcNAc for the transformation in borate solution}

The time-course of GlcNAc transformation was carried out in borate solution with heating as described above. HPLC peaks corresponding to 3, 4 and $\mathbf{5}$ appeared at retention times of 8.5, 5.7 and $5.1 \mathrm{~min}$, respectively (see Experimental section). The time for maximum formation of $\mathbf{3}, \mathbf{4}$ and $\mathbf{5}$ was reached at $\sim 2 \mathrm{~h}$ and their concentrations varied little upon further reaction (Fig. 3a). Compounds 3, 4 and $\mathbf{5}$ were obtained in a total analytical yield of about $80 \%$ and in a ratio of $4.7: 1: 1$, respectively. Compound 3 formed in preference to $\mathbf{4}$ and $\mathbf{5}$, which were produced in equal amount during the entire course of the reaction. GlcNAc and ManNAc are in part epimerized to each other during the reaction process. ${ }^{11-13}$ When ManNAc, instead of GlcNAc, was used as a starting material three products 3, 4 and 5 were generated in a similar ratio and yield (Fig. 3b). Little color was produced at $\mathrm{pH} 7$, but browning occurred at $\mathrm{pH}$ values greater than 8 . Indeed, as the $\mathrm{pH}$ of the reaction increased above 8 there was a significant deepening in this coloration accompanied by an increase in the proportion of 
unknown products other than the three hexofuranoses (data not shown). Thus, the 2-amino sugars rapidly decompose in alkaline solution upon heating to give a multitude of products followed by a browning reaction. ${ }^{8}$ Proximity of the amino group to the anomeric center in 2-amino-sugars confers special properties, which are unusual in the carbohydrate group. ${ }^{14}$ In addition, when GlcNAc was boiled in water as a control experiment, three compounds $\mathbf{3}, \mathbf{4}$ and 5 were also produced, although in extremely low total yields (5\%).

Each of the products 3, 4 and 5 prepared above were reacted again under the same conditions and analyzed by HPLC. Whichever compound was chosen (i.e., 3, 4 or 5), the same products were generated during the reaction. For example, starting with $\mathbf{4}, 85 \%$ of 4 was converted to 3, 5, GlcNAc and ManNAc after $120 \mathrm{~min}$ in a ratio of $4.4: 1.0: 0.7: 0.3$, respectively and their concentrations varied little after prolonged reaction (Fig. 4). These results suggest that the transformation of GlcNAc into 3, $\mathbf{4}$ and $\mathbf{5}$ is a reversible reaction.

The present reaction is proposed to account for a series of hexofuranose transformations as shown in Scheme 1. Despite the complexity of the system, considerable attention has been focused on the reaction of borates with carbohydrates and boron. ${ }^{15}$ Tridentate complexes with borate at 3-, 5- and 6-hydroxys of D-glucofuranose have been studied previously. ${ }^{16}$ Such a formation of borate complex like glucose might facilitate the conversion from the pyranose to the furanose form in borate solution upon heating. Indeed, formation of the complex facilitates abstraction of the 2-hydrogen substituent to give $\mathbf{3}$ followed by dehydration with one water molecule. The resulting $\mathbf{3}$ undergoes a ready epimerization to afford $\mathbf{4}$ and $\mathbf{5}$ in an equal amount, suggesting that they proceed through 2-enol as intermediate by consecutive electron displacement involving intramolecular attack of HO-6 at C-3 in 3. Whichever compound was chosen (i.e., 3, 4, 5 or GlcNAc), the reaction profiles were indistinguishable in the equilibrium state. We therefore conclude that the GlcNAc transformation is a reversible reaction followed by consecutive dehydration and epimerization.

\subsection{Syntheses of furanodictines A (1) and B (2)}

The ability to generate large quantities of $\mathbf{4}$ and $\mathbf{5}$ facilitated the synthesis of 
furanodictines A and B. Compounds 4 and $\mathbf{5}$ were directly converted to furanodictines A (1) and B (2) as shown in Scheme 2. The hydroxy group of $\mathrm{C}_{5}$ in $\mathbf{4}$ and $\mathbf{5}$ were regioselectively esterified with isovaleryl chloride in dry pyridine to complete the total synthesis of $\mathbf{1}$ and $\mathbf{2}$, respectively. The target products $\mathbf{1}$ and $\mathbf{2}$ were purified by chromatography on Silica Gel and ODS column to give yields of 30 and $28 \%$, respectively, based on the amount of $\mathbf{4}$ and $\mathbf{5}$. The structures of these compounds were evaluated by ${ }^{1} \mathrm{H}$ and ${ }^{13} \mathrm{C}$ NMR analyses in $\mathrm{CDCl}_{3}$ solution. In addition, HRESI-MS analysis of $\mathbf{1}$ and $\mathbf{2}$ showed molecular ions at $\mathrm{m} / \mathrm{z} 310.12710$ and 310.12707, respectively, arising from the $[\mathrm{M}+\mathrm{Na}]^{+}$ions. Thus, the target products $\mathbf{1}$ and $\mathbf{2}$ were conveniently synthesized in only a two-step process. The spectral data of synthetic $\mathbf{1}$ and 2 were identical to those of the natural products. ${ }^{1}$

\section{Conclusions}

In conclusion, a total synthesis of furanodictines A and B has been executed via a two-step process starting from readily available GlcNAc. Our process comprises a new synthetic strategy that does not necessitate protection/deprotection. As a result, a facile transformation of GlcNAc into 4 and 5 with a 3,6-anhydro hexofuranose carbon skelton offers a promising approach towards the mass production of furanodictines A and B.

\section{Experimental}

\subsection{General methods}

GlcNAc and isovaleryl chloride were purchased from Sigma-Aldrich (St. Louis, MO). All other reagents were of the highest quality commercially available and were used without further purification.

\subsection{Analytical methods}

HPLC analysis was carried out using a Unison UK-Amino column $(4.6 \times 250 \mathrm{~mm}$, Imtakt $)$ with a JASCO Intelligent system liquid chromatograph and detection at $210 \mathrm{~nm}$. The bound material was eluted with $95 \% \mathrm{CH}_{3} \mathrm{CN}$ at a flow rate of $1.0 \mathrm{~mL} / \mathrm{min}$ at $40^{\circ} \mathrm{C}$. The ESI-MS 
spectra were measured on a JMS-T100LC mass spectrometer. ${ }^{1} \mathrm{H}$ and ${ }^{13} \mathrm{C}$ NMR spectra were recorded on a JEOL JNM-LA 500 spectrometer at $25^{\circ} \mathrm{C}$. Chemical shifts are expressed in $\delta$ relative to sodium 3-(trimethylsilyl) propionate as an external standard.

\subsection{Transformation of GIcNAc into hexofuranoses}

GlcNAc (5.52 g, $25 \mathrm{mmol})$ dissolved in $0.4 \mathrm{M}$ borate buffer $(\mathrm{pH} 7.0,250 \mathrm{~mL})$ was incubated for $2 \mathrm{~h}$ at $100^{\circ} \mathrm{C}$. The mixture was then loaded onto a charcoal-Celite column $(4.5 \times 100 \mathrm{~cm})$ equilibrated with distilled water. Subsequently, the adsorbed portion was eluted with a linear gradient of $0 \%$ to $25 \%$ ethanol in a total volume of $10 \mathrm{~L}$, followed by $25 \%$ ethanol, at a flow rate of $4.7 \mathrm{~mL} / \mathrm{min}$. Fractions $(60 \mathrm{~mL} /$ tube) were collected during the elution of material from the column. The chromatogram identified five distinct peaks. Fractions corresponding to each of the five peaks, F-1 to F-5, were pooled, concentrated and lyophilized. F-1 (fractions 73 86) contained $520 \mathrm{mg}$ as a mixture of GlcNAc and ManNAc in a ratio of $2: 1$, respectively. F-3 (fractions $121 \sim$ 128) was obtained as 2-acetamido-3,6-anhydro-2-deoxy-D-mannofuranose $\quad(5,495 \mathrm{mg}, 9.8 \%)$, which was crystallized from ethanol ${ }^{9}$; $\alpha$-anomer, m. p. $169-170^{\circ} \mathrm{C} ;[\alpha]_{D}^{27}+151.5^{\circ}$ (c 1.0, water); HRESIMS: $m / z 226.06938[\mathrm{M}+\mathrm{Na}]^{+}$(calcd for $\left.\mathrm{C}_{8} \mathrm{H}_{13} \mathrm{~N}_{1} \mathrm{Na}_{1} \mathrm{O}_{5}, 226.06914\right) ;{ }^{1} \mathrm{H}-\mathrm{NMR}\left(\mathrm{D}_{2} \mathrm{O}\right.$, $500 \mathrm{MHz}$, the spectrum was measured after $2 \mathrm{~h}) \alpha$-anomer: $\delta 5.53$ (d, $\left.1 \mathrm{H}, J_{1,2} 5.5 \mathrm{~Hz}, \mathrm{H}-1\right)$, $4.70\left(\mathrm{t}, 1 \mathrm{H}, J_{3,4} 5.5, J_{4,5} 5.5 \mathrm{~Hz}, \mathrm{H}-4\right), 4.65-4.62(1 \mathrm{H}, \mathrm{H}-3), 4.42-4.38(1 \mathrm{H}, \mathrm{H}-5), 4.35$ (t, $1 \mathrm{H}$, $\left.J_{1,2} 5.5, J_{2,3} 5.5 \mathrm{~Hz}, \mathrm{H}-2\right), 3.96-3.89$ (2H, H-6b, H-6a), 2.07 (s, 3H, $\underline{\mathrm{H}}_{3} \mathrm{CONH}$ ); $\beta$-anomer: $\delta$ 5.31 (d, 1H, $\left.J_{1,2} 6.0 \mathrm{~Hz}, \mathrm{H}-1\right), 4.81$ (t, $\left.1 \mathrm{H}, J_{3,4} 4.6, J_{4,5} 4.6 \mathrm{~Hz}, \mathrm{H}-4\right), 4.65-4.62$ (1H, H-3), 4.42-4.38 (1H, H-5), 4.25 (t, 1H, $\left.J_{1,2} 6.0, J_{2,3} 6.0 \mathrm{~Hz}, \mathrm{H}-2\right), 4.02\left(\mathrm{dd}, 1 \mathrm{H}, J_{5,6 \mathrm{~b}} 6.7, J_{6 \mathrm{a}, 6 \mathrm{~b}} 8.4 \mathrm{~Hz}\right.$, H-6b), 3.55 (t, 1H, J5,6a $\left.8.4, J_{6 \mathrm{a}, 6 \mathrm{~b}} 8.4 \mathrm{~Hz}, \mathrm{H}-6 \mathrm{a}\right), 2.05$ (s, 3H, $\underline{\mathrm{H}}_{3} \mathrm{CONH}$ ) $;{ }^{13} \mathrm{C}-\mathrm{NMR}\left(\mathrm{D}_{2} \mathrm{O}\right.$, $500 \mathrm{MHz}) \alpha$-anomer: $\delta 177.0$ ( $\left.\mathrm{CH}_{3} \underline{\mathrm{CONH}}-\right), 98.3$ (C-1), 84.7 (C-4), 83.0 (C-3), 73.95 (C-5), 73.5 (C-6), 57.5 (C-2), 24.4 ( $\left.\underline{\mathrm{CH}}_{3} \mathrm{CONH}-\right)$; $\beta$-anomer: $\delta 177.2$ ( $\left.\mathrm{CH}_{3} \underline{\mathrm{CONH}}-\right), 103.7$ (C-1), 83.5 (C-4), 82.7 (C-3), 74.3 (C-5), 73.90 (C-6), 61.8 (C-2), 24.5 ( CH$\left._{3} \mathrm{CONH}-\right)$. F-4 (fractions 132 139) was obtained as 2-acetamido-3,6-anhydro-2-deoxy-D-glucofuranose (4, $516 \mathrm{mg}$, $10.2 \%$ ), which was crystallized from ethyl acetate. Compound 4 was a mixture of two 
anomers $(\alpha / \beta=2 / 1)$; m.p. $124-125^{\circ} \mathrm{C} ;[\alpha]_{D}^{27}+104.5^{\circ}$ (c 1.0, water); HRESIMS: $m / z 226.07013$ $[\mathrm{M}+\mathrm{Na}]^{+}$(calcd for $\left.\mathrm{C}_{8} \mathrm{H}_{13} \mathrm{~N}_{1} \mathrm{Na}_{1} \mathrm{O}_{5}, 226.06914\right) ;{ }^{1} \mathrm{H}-\mathrm{NMR}\left(\mathrm{D}_{2} \mathrm{O}, 500 \mathrm{MHz}\right) \alpha$-anomer: $\delta$ $5.62\left(\mathrm{~d}, 1 \mathrm{H}, J_{1,2} 5.0 \mathrm{~Hz}, \mathrm{H}-1\right), 4.76\left(\mathrm{t}, 1 \mathrm{H}, J_{3,4} 5.0, J_{4,5} 5.0 \mathrm{~Hz}, \mathrm{H}-4\right), 4.66\left(\mathrm{t}, 1 \mathrm{H}, J_{2,3} 5.0, J_{3,4}\right.$ $5.0 \mathrm{~Hz}, \mathrm{H}-3), 4.33-4.29$ (1H, H-5), 4.27 (t, 1H, $\left.J_{1,2} 5.0, J_{2,3} 5.0 \mathrm{~Hz}, \mathrm{H}-2\right), 4.00$ (dd, 1H, $J_{5,6 \mathrm{~b}}$ 6.5, $\left.J_{6 \mathrm{a}, 6 \mathrm{~b}} 8.5 \mathrm{~Hz}, \mathrm{H}-6 \mathrm{~b}\right), 3.66$ (t, 1H, $\left.J_{5,6 \mathrm{a}} 8.5, J_{6 \mathrm{a}, 6 \mathrm{~b}} 8.5 \mathrm{~Hz}, \mathrm{H}-6 \mathrm{a}\right), 2.06$ (s, 3H, C $\left.\underline{H}_{3} \mathrm{CONH}-\right)$; ß-anomer: $\delta 5.44(1 \mathrm{H}, \mathrm{H}-1), 4.79\left(\mathrm{t}, 1 \mathrm{H}, J_{3,4} 5.0, J_{4,5} 5.0 \mathrm{~Hz}, \mathrm{H}-4\right), 4.52$ (d, 1H, J3,4 $5.0 \mathrm{~Hz}$, H-3), 4.33-4.29 (1H, H-5), 4.18 (1H, H-2), 3.96 (t, 1H, J5,6b 8.0, J6a,6b 8.0 Hz, H-6b), 3.88 (t, $\left.1 \mathrm{H}, J_{5,6 \mathrm{a}} 8.0, J_{6 \mathrm{a}, 6 \mathrm{~b}} 8.0 \mathrm{~Hz}, \mathrm{H}-6 \mathrm{a}\right), 2.02$ (s, 3H, $\left.\underline{\mathrm{H}}_{3} \mathrm{CONH}-\right) ;{ }^{13} \mathrm{C}-\mathrm{NMR}\left(\mathrm{D}_{2} \mathrm{O}, 500 \mathrm{MHz}\right)$

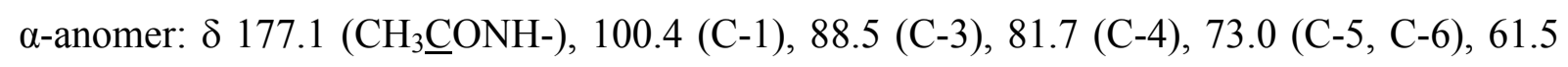
(C-2), 24.56 ( $\left.\underline{\mathrm{CH}}_{3} \mathrm{CONH}-\right)$; $\beta$-anomer: $\delta 176.8$ ( $\mathrm{CH}_{3} \underline{\mathrm{CONH}}$-), 105.2 (C-1), $88.6(\mathrm{C}-3), 85.6$ (C-4), 73.8 (C-6), 73.5 (C-5), 64.7 (C-2), 24.60 ( CH$\left._{3} \mathrm{CONH}-\right)$. F-5 (fractions $162 \sim 197$ ) was obtained as Chromogen I (3) in the form of a syrupy liquid (1.8 g, 36\%). Compound 3 was a mixture of two anomers $(\alpha / \beta=1.6 / 1) ;[\alpha]_{D}^{27}+14.8^{\circ}$ (c 1.0, water); HRESIMS: $m / z 226.06893$ $[\mathrm{M}+\mathrm{Na}]^{+}$(calcd for $\left.\mathrm{C}_{8} \mathrm{H}_{13} \mathrm{~N}_{1} \mathrm{Na}_{1} \mathrm{O}_{5}, 226.06914\right) ;{ }^{1} \mathrm{H}-\mathrm{NMR}\left(\mathrm{D}_{2} \mathrm{O}, 500 \mathrm{MHz}\right) \alpha$-anomer: $\delta$ 6.16 (1H, H-3), 6.04 (1H, H-1), 5.06 (1H, H-4), 3.83-3.57 (3H, H-5, H-6b, H-6a), 2.13 (s, 3H, $\left.\mathrm{CH}_{3} \mathrm{CONH}-\right)$; $\beta$-anomer: $\delta 6.21$ (1H, H-3), 5.99 (1H, H-1), $4.83(1 \mathrm{H}, \mathrm{H}-4), 3.83-3.57$ (3H, H-5, H-6b, H-6a), 2.13 (s, 3H, $\underline{\mathrm{C}}_{3} \mathrm{CONH}-$ ); ${ }^{13} \mathrm{C}-\mathrm{NMR}\left(\mathrm{D}_{2} \mathrm{O}, 500 \mathrm{MHz}\right) \alpha$-anomer: $\delta 176.1$ ( $\mathrm{CH}_{3}$ CONH-), 137.0 (C-2), 112.0 (C-3), 102.2 (C-1), 87.2 (C-4), 76.2 (C-5), 65.2 (C-6), 25.4 ( $\left.\underline{\mathrm{CH}}_{3} \mathrm{CONH}-\right)$; $\beta$-anomer: $\delta 176.1$ ( $\mathrm{CH}_{3} \underline{\mathrm{CONH}}$-), 136.5 (C-2), 112.7 (C-3), 102.0 (C-1), 87.5 (C-4), 76.5 (C-5), 65.1 (C-6), 25.4 ( $\left.\underline{\mathrm{CH}}_{3} \mathrm{CONH}-\right)$.

\subsection{Syntheses of furanodictines A (1) and B (2)}

Compound $4(150 \mathrm{mg}, 0.74 \mathrm{mmol})$ was dissolved in dry pyridine $(5.0 \mathrm{~mL})$ at $0^{\circ} \mathrm{C}$. Isovaleryl chloride (90 $\mu \mathrm{L}, 0.74 \mathrm{mmol}$ ) was added to the solution, and then the mixture was stirred magnetically for $2 \mathrm{~h}$ at room temperature. The reaction was terminated by adding crushed ice. After three extractions with chloroform, the organic layer was washed with saturated sodium bicarbonate solution, water and brine, and then dried over anhydrous sodium sulfate before being concentrated. The reaction products were dissolved in $1.0 \mathrm{~mL}$ of chloroform/methanol 
$=27 / 1$ and then loaded onto a Silica Gel $60 \mathrm{~N}$ column $(2.0 \times 40 \mathrm{~cm})$. The column was developed with the same solvent at a flow rate of $10 \mathrm{~mL} / \mathrm{min}$ and a fraction size of 15 $\mathrm{mL} /$ tube. An aliquot from fractions $36 \sim 50$ were concentrated and dissolved in $1.0 \mathrm{~mL}$ of $\mathrm{H}_{2} \mathrm{O}$ and then loaded onto a ODS column $(1.5 \times 30 \mathrm{~cm})$. The bound compound was eluted with a linear gradient of 0 to $30 \%$ methanol in a total volume of $1.2 \mathrm{~L}$ at a flow rate of 2.0 $\mathrm{mL} / \mathrm{min}$, and a fraction size of $15 \mathrm{~mL} /$ tube. Fractions $70 \sim 76$ were pooled and concentrated. Furanodictine A (1) was obtained in a total yield of $30 \%(64 \mathrm{mg})$ as a colorless oil. Compound 1 was a mixture of two anomers $(\alpha / \beta=6.8 / 1) ; R_{f}=0.20($ EtOAc $) ;[\alpha]_{D}^{25}+116.3^{\circ}(c$ 0.8, $\mathrm{CHCl}_{3}$ ); HRESIMS: $m / z 310.12710[\mathrm{M}+\mathrm{Na}]^{+}$(calcd for $\mathrm{C}_{13} \mathrm{H}_{21} \mathrm{~N}_{1} \mathrm{Na}_{1} \mathrm{O}_{6}, 310.12666$ ); ${ }^{1} \mathrm{H}-\mathrm{NMR}\left(\mathrm{CDCl}_{3}, 500 \mathrm{MHz}\right) \alpha$-anomer: $\delta 6.40\left(\mathrm{~d}, 1 \mathrm{H}, J_{\mathrm{NH}, 2} 7.5 \mathrm{~Hz}, \mathrm{NH}\right), 5.50\left(\mathrm{~d}, 1 \mathrm{H}, J_{1,2} 4.5\right.$ Hz, H-1), 4.95 (ddd, 1H, $\left.J_{4,5} 5.5, J_{5,6 \mathrm{a}} 8.0, J_{5,6 \mathrm{~b}} 6.0 \mathrm{~Hz}, \mathrm{H}-5\right), 4.84$ (t, $1 \mathrm{H}, J_{3,4} 5.5, J_{4,5} 5.5 \mathrm{~Hz}$, H-4), 4.52 (dd, 1H, J2,3 4.0, $\left.J_{3,4} 5.5 \mathrm{~Hz}, \mathrm{H}-3\right), 4.33$ (m, 1H, H-2), 4.01 (dd, 1H, $J_{5,6 \mathrm{~b}} 6.0, J_{6 \mathrm{a}, 6 \mathrm{~b}}$ $9.0 \mathrm{~Hz}, \mathrm{H}-6 \mathrm{~b}), 3.77$ (dd, 1H, $\left.J_{5,6 \mathrm{a}} 8.0, J_{6 \mathrm{a}, 6 \mathrm{~b}} 9.0 \mathrm{~Hz}, \mathrm{H}-6 \mathrm{a}\right), 2.25-2.17\left(2 \mathrm{H}, \mathrm{C}_{2}\right), 2.10-2.02(1 \mathrm{H}$, $\mathrm{C} \underline{\mathrm{H}}), 1.99$ (s, 3H, $\left.\underline{\mathrm{C}}_{3} \mathrm{CONH}-\right), 0.92$ (d, 6H, $\underline{\mathrm{H}}_{3}$ ); $\beta$-anomer: $\delta 5.50$ (br.s, $1 \mathrm{H}, \mathrm{N} \underline{\mathrm{H}}$ ), 5.22 (br.s, $1 \mathrm{H}, \mathrm{H}-1$ ), 5.04 (ddd, $\left.1 \mathrm{H}, J_{4,5} 5.0, J_{5,6 \mathrm{a}} 6.0, J_{5,6 \mathrm{~b}} 4.5 \mathrm{~Hz}, \mathrm{H}-5\right), 4.87$ (t, $1 \mathrm{H}, J_{3,4} 5.0, J_{4,5} 5.0 \mathrm{~Hz}$, H-4), 4.38 (1H, H-3), 4.17 (m, 1H, H-2), 4.09 (dd, 1H, J5,6b 4.5, J6a,6b 9.5 Hz, H-6b), 3.89 (dd, $\left.1 \mathrm{H}, J_{5,6 \mathrm{a}} 6.0, J_{6 \mathrm{a}, 6 \mathrm{~b}} 9.5 \mathrm{~Hz}, \mathrm{H}-6 \mathrm{a}\right), 2.25-2.17\left(2 \mathrm{H}, \underline{\mathrm{C}}_{2}\right), 2.10-2.02(1 \mathrm{H}, \mathrm{C} \underline{\mathrm{H}}), 1.96(\mathrm{~s}, 3 \mathrm{H}$, $\left.\mathrm{CH}_{3} \mathrm{CONH}-\right), 0.92\left(\mathrm{~d}, 6 \mathrm{H}, \underline{\mathrm{CH}}_{3}\right) ;{ }^{13} \mathrm{C}-\mathrm{NMR}\left(\mathrm{CDCl}_{3}, 500 \mathrm{MHz}\right) \alpha$-anomer: $\delta 172.6\left(\mathrm{C}^{\prime}-1\right)$, $170.8\left(\mathrm{CH}_{3} \underline{\mathrm{CONH}}-\right), 98.0$ (C-1), 86.9 (C-3), 77.7 (C-4), 72.2 (C-5), 68.6 (C-6), 58.6 (C-2), 42.9 (C'-2), 25.6 (C'-3), 23.0 ( CH$_{3} \mathrm{CONH}-$ ), 22.3 (C'-4, C'-5); $\beta$-anomer: $\delta 172.8$ (C'-1), $170.6\left(\mathrm{CH}_{3} \underline{\mathrm{CONH}}-\right), 103.4$ (C-1), 86.5 (C-3), 81.6 (C-4), 73.1 (C-5), 70.6 (C-6), 61.2 (C-2), 42.8 (C'-2), 25.4 (C'-3), $22.9\left(\underline{\mathrm{CH}}_{3} \mathrm{CONH}-\right), 22.3$ (C’-4, C'-5). Furanodictine B (2) was obtained in a similar manner with a total yield $28 \%(59 \mathrm{mg})$ as a colorless oil from compound 5 and isovaleryl chloride. Compound 2 was a mixture of two anomers $(\alpha / \beta=1 / 2.5) ; R_{f}=0.21$ (EtOAc); $[\alpha]_{D}^{25}+103.9^{\circ}\left(c\right.$ 0.8, $\mathrm{CHCl}_{3}$ ); HRESIMS: $m / z 310.12707[\mathrm{M}+\mathrm{Na}]^{+}$(calcd for $\left.\mathrm{C}_{13} \mathrm{H}_{21} \mathrm{~N}_{1} \mathrm{Na}_{1} \mathrm{O}_{6}, 310.12666\right) ;{ }^{1} \mathrm{H}-\mathrm{NMR}\left(\mathrm{CDCl}_{3}, 500 \mathrm{MHz}\right) \alpha$-anomer: $\delta 6.16\left(\mathrm{~d}, 1 \mathrm{H}, J_{\mathrm{NH}, 2} 7.5\right.$ $\mathrm{Hz}, \mathrm{N} \underline{\mathrm{H}}), 5.17$ (d, 1H, $\left.J_{1,2} 4.0 \mathrm{~Hz}, \mathrm{H}-1\right), 5.10$ (td, $\left.1 \mathrm{H}, J_{4,5} 5.0, J_{5,6 \mathrm{a}} 5.5, J_{5,6 \mathrm{~b}} 5.5 \mathrm{~Hz}, \mathrm{H}-5\right), 4.90$ (t, 1H, $\left.J_{3,4} 5.0, J_{4,5} 5.0 \mathrm{~Hz}, \mathrm{H}-4\right), 4.55$ (dd, 1H, $\left.J_{2,3} 6.5, J_{3,4} 5.0 \mathrm{~Hz}, \mathrm{H}-3\right), 4.15$ (m, 1H, H-2), 
$4.00\left(\mathrm{dd}, 1 \mathrm{H}, J_{5,6 \mathrm{~b}} 5.5, J_{6 \mathrm{a}, 6 \mathrm{~b}} 9.5 \mathrm{~Hz}, \mathrm{H}-6 \mathrm{~b}\right), 3.76$ (dd, 1H, $\left.J_{5,6 \mathrm{a}} 5.5, J_{6 \mathrm{a}, 6 \mathrm{~b}} 9.5 \mathrm{~Hz}, \mathrm{H}-6 \mathrm{a}\right)$, 2.25-2.16 (2H, $\left.\mathrm{C}_{2}\right), 2.09-2.01(1 \mathrm{H}, \mathrm{C} \underline{\mathrm{H}}), 1.98$ (s, 3H, $\left.\mathrm{C}_{3} \mathrm{CONH}-\right), 0.92-0.90\left(6 \mathrm{H}, \mathrm{C}_{3}\right)$; $\beta$-anomer: $\delta 6.16\left(\mathrm{~d}, 1 \mathrm{H}, J_{\mathrm{NH}, 2} 7.5 \mathrm{~Hz}, \mathrm{~N} \underline{\mathrm{H}}\right), 5.32$ (br.s, $\left.1 \mathrm{H}, \mathrm{H}-1\right), 5.04$ (td, $1 \mathrm{H}, J_{4,5} 5.5, J_{5,6 \mathrm{a}} 6.5$, $\left.J_{5,6 \mathrm{~b}} 6.5 \mathrm{~Hz}, \mathrm{H}-5\right), 4.86$ (dd, 1H, $\left.J_{3,4} 5.0, J_{4,5} 5.5 \mathrm{~Hz}, \mathrm{H}-4\right), 4.47$ (1H, H-3), 4.37 (m, 1H, H-2), $4.03\left(\mathrm{dd}, 1 \mathrm{H}, J_{5,6 \mathrm{~b}} 6.5, J_{6 \mathrm{a}, 6 \mathrm{~b}} 9.0 \mathrm{~Hz}, \mathrm{H}-6 \mathrm{~b}\right), 3.92$ (dd, 1H, J5,6a $\left.6.5, J_{6 \mathrm{a}, 6 \mathrm{~b}} 9.0 \mathrm{~Hz}, \mathrm{H}-6 \mathrm{a}\right)$, 2.25-2.16 (2H, $\left.\underline{\mathrm{C}}_{2}\right), 2.09-2.01(1 \mathrm{H}, \mathrm{C} \underline{\mathrm{H}}), 1.99$ (s, 3H, $\left.\underline{\mathrm{H}}_{3} \mathrm{CONH}-\right), 0.92-0.90\left(6 \mathrm{H}, \mathrm{C}_{3}\right)$; ${ }^{13} \mathrm{C}-\mathrm{NMR}\left(\mathrm{CDCl}_{3}, 500 \mathrm{MHz}\right) \alpha$-anomer: $\delta 172.2$ (C'-1), $171.0\left(\mathrm{CH}_{3} \mathrm{CONH}-\right), 103.5$ (C-1), 80.3 (C-3), 79.6 (C-4), 72.8 (C-5), 70.7 (C-6), 58.9 (C-2), 42.9 (C'-2), 25.6 (C'-3), 23.1 ( CH$_{3} \mathrm{CONH}-$ ), 22.3 (C'-4, C'-5); $\beta$-anomer: $\delta 172.3$ (C'-1), 170.3 ( $\mathrm{CH}_{3} \underline{\mathrm{CONH}}-$ ), 96.4 (C-1), 80.7 (C-3), 80.6 (C-4), 73.4 (C-5), 69.7 (C-6), 54.6 (C-2), 42.8 (C'-2), 25.5 (C'-3), 23.0 ( $\left.\mathrm{CH}_{3} \mathrm{CONH}-\right), 22.3$ (C'-4, C'-5).

\section{Acknowledgements}

This work was supported by the Programme for Promotion of Basic and Applied Researches for Innovations in Bio-oriented Industry in Japan. 


\section{References}

1. Kikuchi, H.; Saito, Y.; Komiya, J.; Takaya, Y.; Honma, S.; Nakahata, N.; Ito, A.; Oshima, Y. J. Org. Chem. 2001, 66, 6982-6987.

2. Maeda, Y.; Inoue, K.; Takeuchi, I. Dictyostelium A Model System for Cell and Development Biology. Frontiers Science Series No. 21; Universal Academy.; Tokyo, 1997.

3. Yoda, H.; Suzuki, Y.; Takabe, K. Tetrahedron Lett. 2004, 45, 1599-1601.

4. Mereyala, H. B.; Baseeruddin, M.; Koduru, S. R. Tetrahedron: Asymmetry 2004, 15, $3457-3460$.

5. Matsuura, D.; Mitsui, T.; Sengoku, T.; Takahashi, M.; Yoda, H. Tetrahedron 2008, 64, 11686-11696.

6. Morgan, W. T. J.; Elson, L. A. Biochem. J. 1934, 28, 988-995.

7. Kuhn, R.; Kruger, G. Chem. Ber. 1956, 89, 1473-1486.

8. Beau, J. M.; Rollin, P.; Sinay, P. Carbohydr. Res. 1977, 53, 187-195.

9. Derevitskaya, V. A.; Likhosherstov, L. M.; Schennikov, V. A.; Kochetkov, N. K. Carbohydr. Res. 1971, 20, 285-291.

10. Pravdic, N.; Fletcher Jr, H. G. Carbohydr. Res. 1971, 19, 339-352.

11. Coxon, B.; Hough, L. J. Chem. Soc. 1961, 1577-1579.

12. Roden, L.; Jin, J.; Yu, H.; Campbell, P. Glycobiology 1995, 5, 167-173.

13. Yamaguchi, S.; Ohnishi, J.; Maru, I.; Ohta, Y. Trends Glycosci. Glycotechnol. 2006, 18, 245-252.

14. Coxon, B.; Hough, L. J. Chem. Soc. 1961, 1577-1579.

15. Ferrier, R. J. Adv. Carbohydr. Chem. Biochem. 1978, 35, 31-80. Academic. Press. New. York.

16. Foster, A. B.; Stacey, M. J. Chem. Soc. 1955, 1778-1781. 


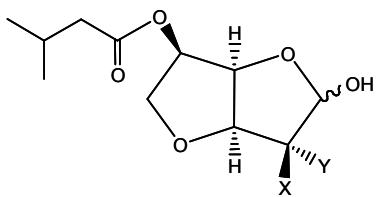

$\mathrm{X}=\mathrm{H}, \mathrm{Y}=$ NHAc: furanodictine $\mathrm{A}(\mathbf{1})$

$\mathrm{X}=\mathrm{NHAc}, \mathrm{Y}=\mathrm{H}$ : furanodictine $\mathrm{B}(\mathbf{2})$

Figure 1. Structures of Furanodictines A and B. 


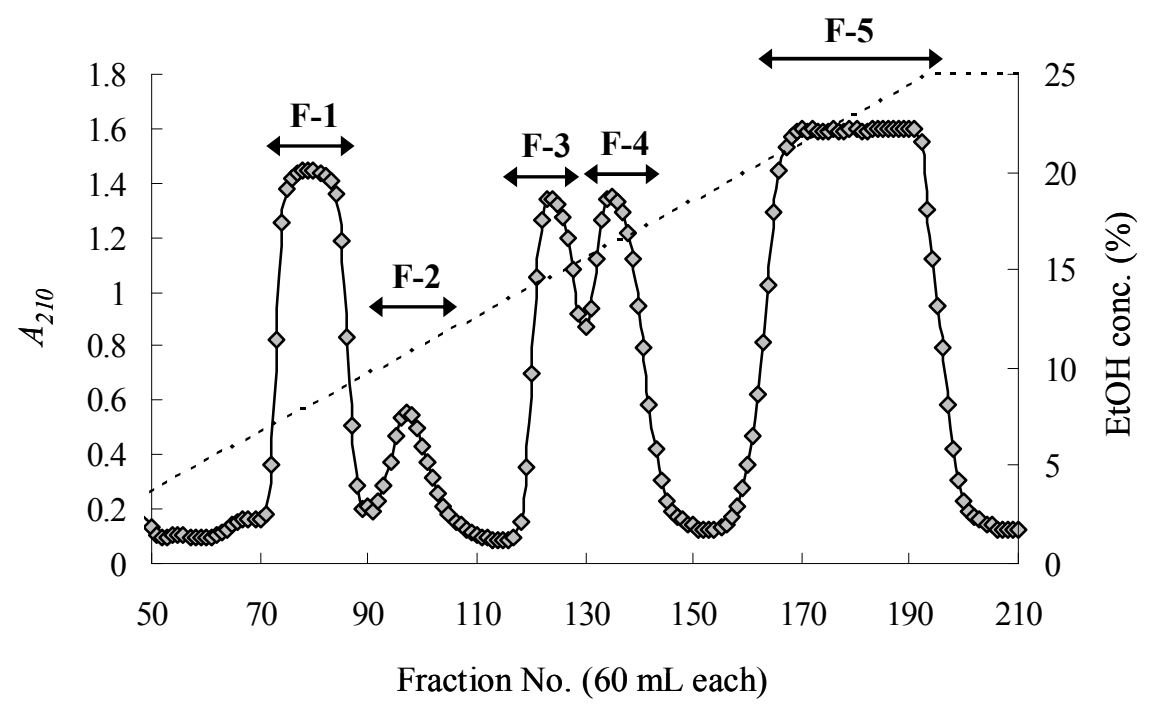

Figure 2. Charcoal-Celite chromatography of products formed from GlcNAc transformation. Dotted line, concentration of ethanol in gradient elution (\%). 

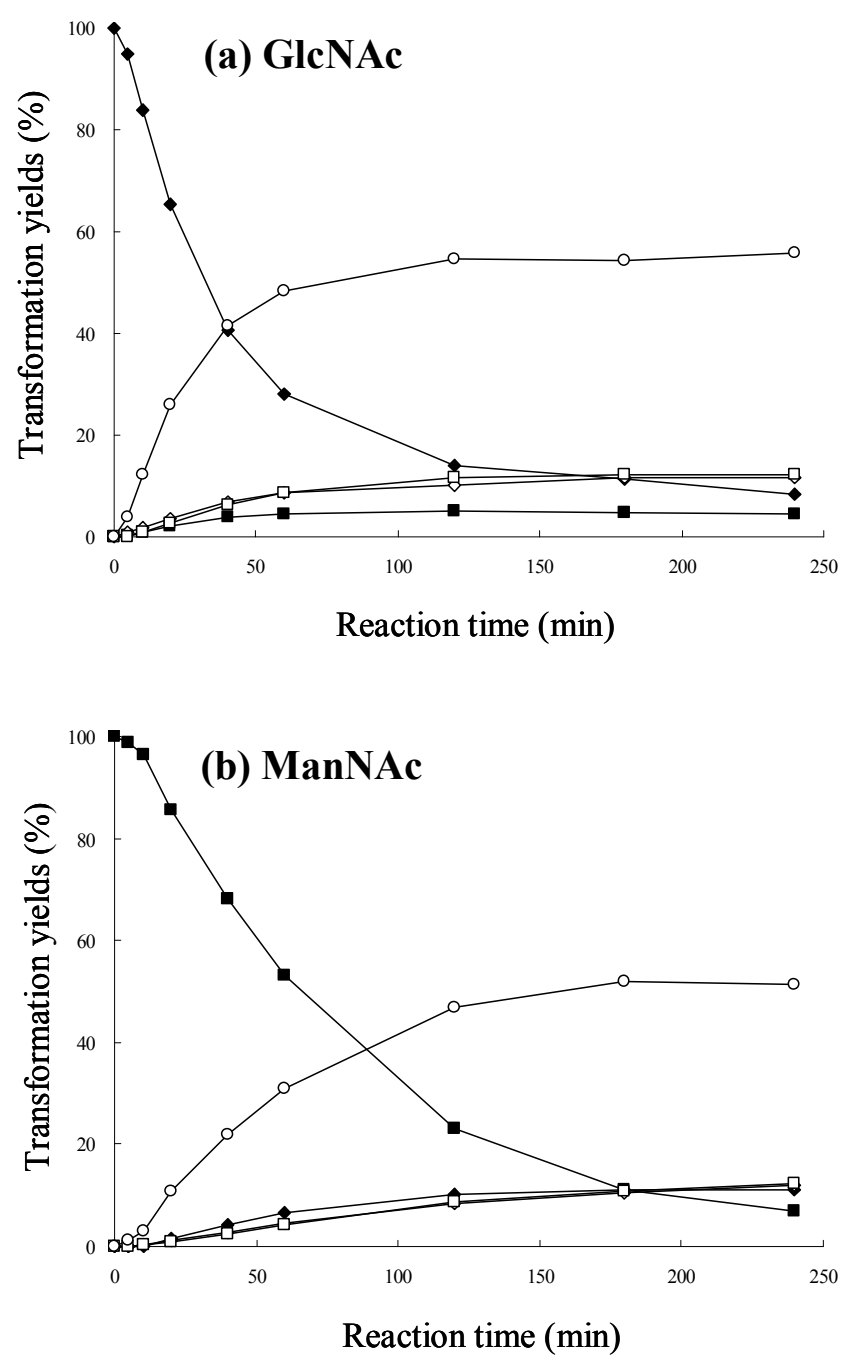

Figure 3. HPLC analyses of GlcNAc/ManNAc on transformation in borate solution (pH 7.0) upon heating. Time-course of products $\mathbf{3}, \mathbf{4}$ and $\mathbf{5}$ on transformation formed from GlcNAc/ManNAc. (a) GlcNAc. (b) ManNAc. Filled diamond, GlcNAc; filled square, ManNAc; open circle, $\mathbf{3}$; open diamond, 4; open square, 5. 


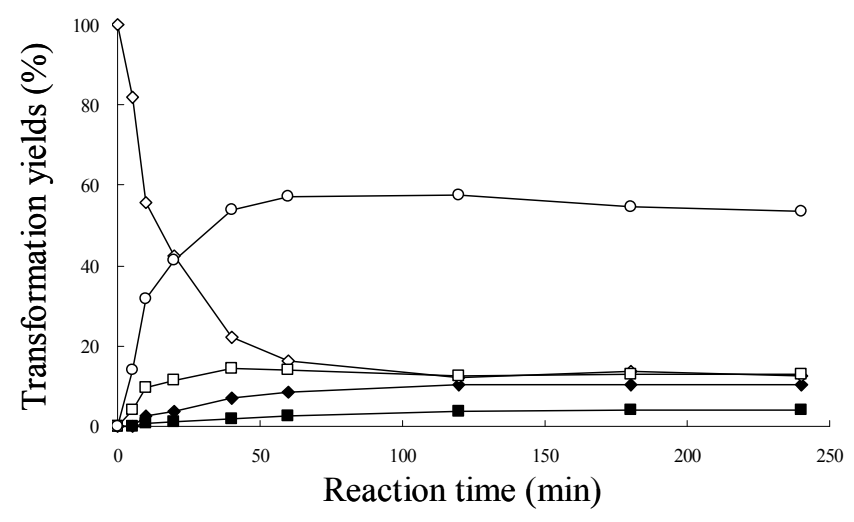

Figure 4. Time-course of products 3, 5, GlcNAc and ManNAc on transformation in borate solution ( $\mathrm{pH}$ 7.0) upon heating formed from 4. Filled diamond, GlcNAc; filled square, ManNAc; open circle, 3; open diamond, 4; open square, 5. 


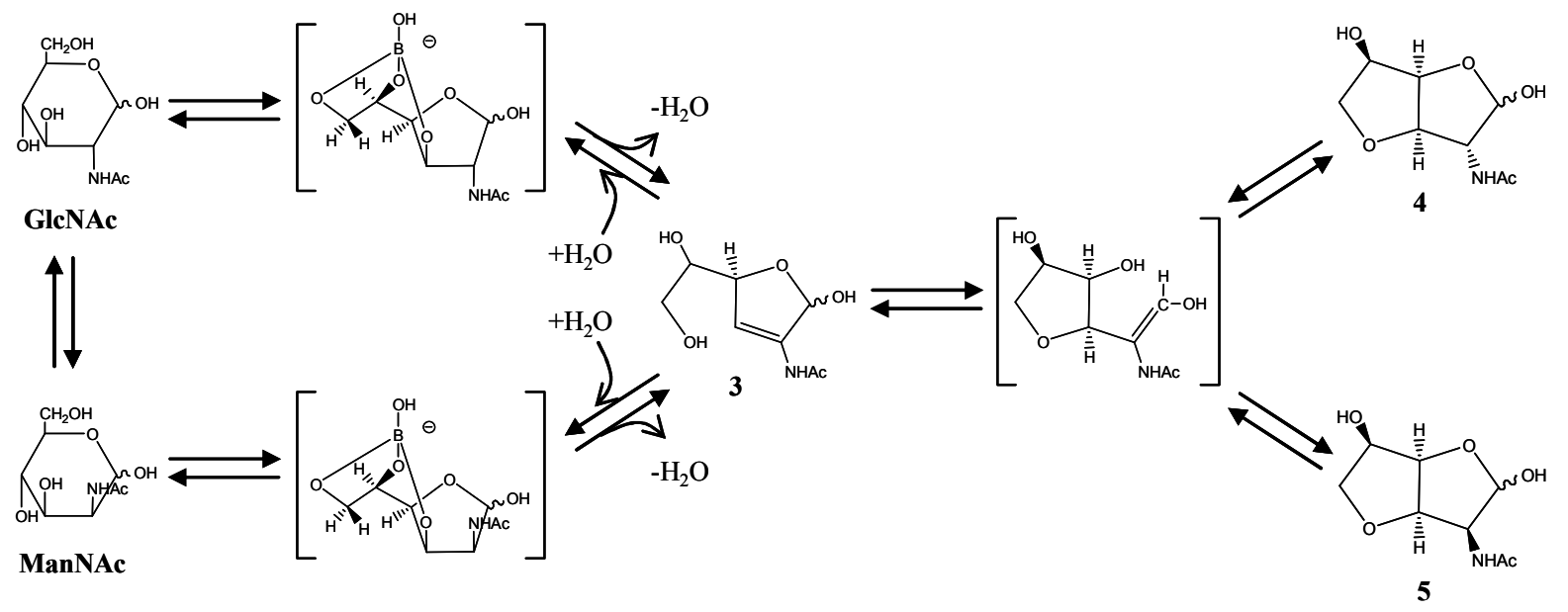

Scheme 1. Proposed mechanism of transformation of GlcNAc/ManNAc into 3, 4 and $\mathbf{5}$ in borate solution upon heating. 


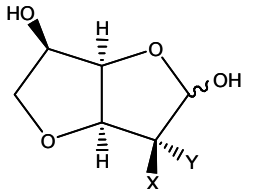

$\mathrm{X}=\mathrm{H}, \mathrm{Y}=\mathrm{NHAc}(\mathbf{4})$

$\mathrm{X}=\mathrm{NHAc}, \mathrm{Y}=\mathrm{H}(\mathbf{5})$
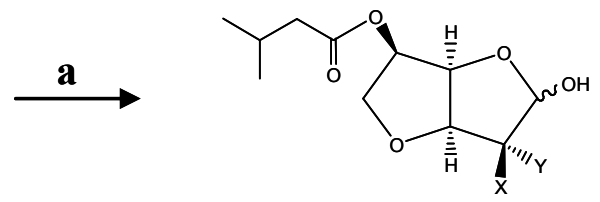

$\mathrm{X}=\mathrm{H}, \mathrm{Y}=$ NHAc: furanodictine A (1)

$\mathrm{X}=\mathrm{NHAc}, \mathrm{Y}=\mathrm{H}$ : furanodictine $\mathrm{B}$ (2)

Scheme 2. Syntheses of Furanodictines A and B. Reagents and conditions: (a) dry pyridine, isovaleryl chloride, $25^{\circ} \mathrm{C}, 2 \mathrm{~h}, 30 \%$ (1), $28 \%$ (2). 